\title{
Extraction of Desired Signal Based on AR Model with Its Application to Atrial Activity Estimation in Atrial Fibrillation
}

\author{
Gang Wang, ${ }^{1,2}$ Ni-ni Rao, ${ }^{1}$ Simon J. Shepherd, ${ }^{2}$ and Clive B. Beggs ${ }^{2}$ \\ ${ }^{1}$ School of life Science and Technology, University of Electronic Science and Technology of China, Chengdu 610054, China \\ ${ }^{2}$ Medical Biophysics Group, School of Engineering, Design and Technology, University of Bradford, BD7 1DP Bradford, UK
}

Correspondence should be addressed to Ni-ni Rao, cliu@uestc.edu.cn

Received 28 July 2007; Revised 15 February 2008; Accepted 23 April 2008

Recommended by Aníbal Figueiras-Vidal

The use of electrocardiograms (ECGs) to diagnose and analyse atrial fibrillation (AF) has received much attention recently. When studying AF, it is important to isolate the atrial activity (AA) component of the ECG plot. We present a new autoregressive (AR) model for semiblind source extraction of the AA signal. Previous researchers showed that one could extract a signal with the smallest normalized mean square prediction error (MSPE) as the first output from linear mixtures by minimizing the MSPE. However the extracted signal will be not always the desired one even if the AR model parameters of one source signal are known. We introduce a new cost function, which caters for the specific AR model parameters, to extract the desired source. Through theoretical analysis and simulation we demonstrate that this algorithm can extract any desired signal from mixtures provided that its AR parameters are first obtained. We use this approach to extract the AA signal from 12-lead surface ECG signals for hearts undergoing AF. In our methodology we roughly estimated the AR parameters from the fibrillatory wave segment in the V1 lead, and then used this algorithm to extract the AA signal. We validate our approach using real-world ECG data.

Copyright $\odot 2008$ Gang Wang et al. This is an open access article distributed under the Creative Commons Attribution License, which permits unrestricted use, distribution, and reproduction in any medium, provided the original work is properly cited.

\section{INTRODUCTION}

In recent years, there has been considerable interest in the electrical and physiological mechanisms associated with atrial fibrillation (AF) [1-4]. $\mathrm{AF}$ is a relatively common arrhythmia, which occurs when the atria depolarize repeatedly in an irregular uncontrolled manner. During AF, electrical discharges come from other parts of the atria, rather than solely from the sinoatrial (SA) node. These abnormal irregular discharges are very rapid and result in ineffective contraction of the atria, so that they quiver rather than beat as a unit. This reduces the ability of the atria to discharge blood into the ventricles, thus impairing the performance of the heart. AF is of clinical importance because it is associated with an increased risk of morbidity and mortality, particularly amongst the elderly who are more prone to this condition.

$\mathrm{AF}$ is generally diagnosed by visual inspection of the surface electrocardiogram (ECG). On an ECG plot, AF is characterized by a plot which has no clear P-wave, only a fine apparently disorganized oscillation (known as a fibrillatory or F-wave), and a ventricular response which is fast and irregular. When studying $\mathrm{AF}$ it is important to isolate the atrial activity (AA) component of the ECG plot. However, because the electrical activity of the ventricles is of greater amplitude of the atria, it is difficult to identify the atrial component. Several methods have been developed to address this problem. Some are based on average beat subtraction (ABS), which assumes that the AA is uncoupled with the ventricular activity (VA). This approach uses an average of the ventricular QRST complexes which is then subtracted from the wave to determine AA [5]. However, this approach is limited by the small number of VA average templates available for general VA approximation [3]. Recently, methods which utilize blind source separation (BSS) have been developed for extracting AA signals from ECG plots [6-10]. The objective of this approach is to recover the unknown source signal from the mixture without knowing the mixing channels. While BSS appears promising, it has the drawback that it requires considerable computational power. In an attempt to address this issue, we undertook a study using a blind source extraction (BSE) approach to solve the problem. BSE is a powerful technique related to BSS [11, 12], which has become one of the major research areas in signal 
processing. The approach taken in BSE is to sequentially extract small subsets from the source signal, which are independent from each other, but linearly combined in the observations. Compared with BSS, BSE requires a much lower computational load and is therefore less expensive. As a result it has received considerable interest and has been used in various fields such as biomedical signal processing [13] and speech processing $[11,12]$.

Many algorithms designed for BSE have been proposed, including those employing high-order statistics (HOS) [14, 15] and those employing second-order statistics (SOS) [13, 15-19]. Cichocki and Amari [11] give a comprehensive overview of these algorithms. However, these algorithms are generally designed to extract signals in a specific order [19], or extract special signals, such as fetal ECG [13] and fMRI data [14], and they do not always work well when dealing with an AA signal. AA sources have a main peak between $3.5-10 \mathrm{~Hz}$ [10], where the observed ECG plot has two or more apparently random peaks. So it is not possible to estimate directly distinct periods in the signal. Consequently, algorithms based on periodic structure [13, 16, 17, 19] will fail. Moreover, the AA wave is not stationary, so it is difficult for a constrained independent component analysis (ICA) algorithm [14] to select the referent signal and thus extract the AA data.

Given that AA signals exhibit a narrowband spectrum $[20,21]$ with main frequencies between $3.5-10 \mathrm{~Hz}$ [8], in our study we came to the conclusion that the linear predictor or autoregressive (AR) model could be regarded as stable. Moreover, we determined that it was possible to estimate the AR parameters from the fibrillatory wave segment of the surface ECG plot. While BSE algorithms $[17,19]$ employing a linear predictor usually minimize the normalized mean square prediction error (MSPE), they may not extract the specific signal, even though its AR parameters may be known. In this paper, we therefore introduced a new cost function, which caters for the specific AR model parameters, and propose an algorithm based on eigenvalue decomposition (EVD) to extract the desired signal. In the paper, we validate this algorithm and illustrate how it can be used to estimate the AR parameters of the AA wave signal. We also summarize techniques that can be used to extract AA signals based on a BSE approach.

\section{LIMITATION OF MSPE}

In BSE, we observe an $m$-dimensional stochastic signal vector $\mathbf{x}$ that is regarded as the linear mixture of an $m$ dimensional zero-mean and unit-variance vector $\mathbf{s}$, that is, $\mathbf{x}=\mathbf{A s}$, where $\mathbf{A}$ is an unknown mixing matrix. The goal of BSE is to find a demixing vector $w$ such that $y=\mathbf{w}^{T} \mathbf{x}=$ $\mathbf{w}^{T}$ As is an estimated source signal up to a scalar. To make algorithm more robust and faster, prewhitening is often used to transform the observed signals $x$ to $\mathbf{x}=\mathbf{V} \mathbf{x}$, such that $E\left\{\underline{\mathbf{x x}}^{T}\right\}=\mathbf{V A} E\left\{\underline{\mathbf{s s}}^{T}\right\} \mathbf{A}^{T} \mathbf{V}^{T}=\mathbf{V A A}^{T} \mathbf{V}^{T}=\mathbf{I}$, where $\mathbf{V}$ is a prewhitening matrix. Therefore, for convenience, in this paper we will assume that $\mathbf{x}$ has been prewhitened in the following, that is, $E\left\{\mathbf{x x} \mathbf{x}^{T}\right\}=\mathbf{I}, \mathbf{A}$ is an orthogonal matrix, and $\mathbf{w}^{T} \mathbf{w}=1$.
If we assume that the sources are not correlated with each other and have different temporal structures, then the following relations are satisfied:

$$
E\left\{s_{i}(n) s_{j}(n-\tau)\right\}=0, \quad \forall i \neq j, 0 \leq \tau \leq p,
$$

where $p$ is the length of the linear predictor or AR model. Then the instantaneous prediction error (PE) denoted by $e(n)$ is as follows:

$$
\begin{aligned}
e(n) & =y(n)-\mathbf{b}^{T} \mathbf{Y}(n), \\
\mathbf{b} & =\left[b_{1}, b_{2}, \ldots, b_{P}\right]^{T}, \\
\mathbf{Y}(n) & =[y(n-1), y(n-2), \ldots, y(n-p)]^{T}, \\
y(n) & =\mathbf{w}^{T} \mathbf{x}(n), \\
\mathbf{x}(n) & =\left[x_{1}(n), x_{2}(n), \ldots, x_{m}(n)\right]^{T},
\end{aligned}
$$

where $\mathbf{b}$ is the AR parameters of a desired signal.

It has been shown by Liu et al. [17, 19] that source signals can be extracted successfully by minimizing the normalized MSPE $E\left\{e^{2}(n)\right\}$ as long as they have different temporal structures. The corresponding cost function is the normalized MSPE $E\left\{e^{2}(n)\right\} / E\left\{y^{2}(n)\right\}$. As mentioned above, we assume here that $\mathbf{x}$ has been prewhitened and thus that the output power of the demixing vector $E\left\{y^{2}(n)\right\}$ is unity. Therefore, the cost function can be set as $E\left\{e^{2}(n)\right\}$.

If we know the AR model of the desired source signal, $s_{1}(n)$, then we can easily identify its linear predictor coefficients. The PE of the extracted signal therefore can be written as

$$
\begin{aligned}
e(n)= & y(n)-\mathbf{b}^{T} \mathbf{Y}(n) \\
= & \mathbf{w}^{T} \mathbf{x}(n)-\left[b_{1}, b_{2}, \ldots, b_{P}\right] \\
& \times\left[\mathbf{w}^{T} \mathbf{x}(n-1), \mathbf{w}^{T} \mathbf{x}(n-2), \ldots, \mathbf{w}^{T} \mathbf{x}(n-p)\right]^{T} \\
= & \mathbf{w}^{T} \mathbf{x}(n)-\mathbf{w}^{T} \sum_{i=1}^{p} b_{i} \mathbf{x}(n-i) \\
= & \mathbf{w}^{T}\left(\mathbf{x}(n)-\sum_{i=1}^{p} b_{i} \mathbf{x}(n-i)\right) .
\end{aligned}
$$

Denote

$$
z(n)=\left(\mathbf{x}(n)-\sum_{i=1}^{p} b_{i} \mathbf{x}(n-i)\right)
$$

The normalized MSPE is

$$
\frac{E\left\{e^{2}(n)\right\}}{E\left\{y^{2}(n)\right\}}=\frac{E\left\{\mathbf{w}^{T} z(n) z^{T}(n) \mathbf{w}\right\}}{E\left\{\mathbf{w}^{T} \mathbf{x}(n) \mathbf{x}^{T}(n) \mathbf{w}\right\}}=\frac{\mathbf{w}^{T} E\left\{z(n) z^{T}(n)\right\} \mathbf{w}}{\mathbf{w}^{T} \mathbf{w}} .
$$


Moreover,

$$
\begin{aligned}
& E\left\{z(n) z^{T}(n)\right\} \\
& =E\left\{\left(\mathbf{x}(n)-\sum_{i=1}^{p} b_{i} \mathbf{x}(n-i)\right)\left(\mathbf{x}(n)-\sum_{i=1}^{p} b_{i} \mathbf{x}(n-i)\right)^{T}\right\} \\
& =E\left\{\left(\mathbf{A s}(n)-\sum_{i=1}^{p} b_{i} \mathbf{A s}(n-i)\right)\left(\mathbf{A} \mathbf{s}(n)-\sum_{i=1}^{p} b_{i} \mathbf{A s}(n-i)\right)^{T}\right\} \\
& =\mathbf{A} E\left\{\left(\mathbf{s}(n)-\sum_{i=1}^{p} b_{i} \mathbf{s}(n-i)\right)\left(\mathbf{s}(n)-\sum_{i=1}^{p} b_{i} \mathbf{s}(n-i)\right)^{T}\right\} \mathbf{A}^{T} .
\end{aligned}
$$

Denote

$$
\mathbf{R}_{p}=E\left\{\left(\mathbf{s}(n)-\sum_{i=1}^{p} b_{i} \mathbf{s}(n-i)\right)\left(\mathbf{s}(n)-\sum_{i=1}^{p} b_{i} \mathbf{s}(n-i)\right)^{T}\right\}
$$

which is a diagonal matrix with the help of expression (1), and whose diagonal element $\mathbf{R}_{p}(j, j)$ equals the $\operatorname{MSPEE}\left\{\left\{e_{j}^{2}(\mathrm{n})\right\}\right\}$ of the corresponding source signal:

$$
\begin{aligned}
\mathbf{R}_{p}(j, j) & =E\left\{\left\{e_{j}^{2}(n)\right\}\right\} \\
& =E\left\{\left(s_{j}(n)-\mathbf{b}^{T} \mathbf{S}_{j}(n)\right)^{2}\right\}, \quad j=1,2, \ldots, m . \\
S_{j}(n) & =\left[s_{j}(n-1), s_{j}(n-2), \ldots, s_{j}(n-p)\right]^{T} .
\end{aligned}
$$

Then the normalized MSPE becomes

$$
\frac{E\left\{e^{2}(n)\right\}}{E\left\{y^{2}(n)\right\}}=\frac{\mathbf{w}^{T} \mathbf{A} \mathbf{R}_{p} \mathbf{A}^{T} \mathbf{w}}{\mathbf{w}^{T} \mathbf{w}}
$$

which implies that the minimization of the normalized MSPE under the constraint $\mathbf{w}^{T} \mathbf{w}=1$ is equivalent to finding the eigenvector corresponding to the minimal eigenvalue of the real symmetric matrix $E\left\{z(n) z^{T}(n)\right\}$. Moreover, since A is orthogonal and $\mathbf{R}_{p}$ is diagonal matrix, theoretically the minimal eigenvalue is equivalent to the minimum of the diagonal elements $\mathbf{R}_{p}(j, j)(\mathrm{j}=1,2, \ldots, m)$. Thus we can conclude that the first extracted signal by this method is the one whose MSPE is minimal for a given AR parameter.

However, this argument may not be as straight forward as it seems, because it raises interesting questions as to whether or not the desired source signal has the minimum normalized MSPE among its sources. If, for example, we consider the benchmarks $s_{1}, s_{2}, s_{3}$, and $s_{4}$ utilized in [19], which are shown in Figure 1 (which can be found in the file Abio7.mat provided in the ICALAB toolbox with book [11]), it is possible to calculate using (8) the MSPEs of the four signals for the given AR parameter of the different sources. The results of this analysis are summarized in Table 1 for $p=10$ and in Table 2 for $p=20$, where the minimum data in each row are accented with bold cases. In Table 1 we can see that $s_{3}, s_{4}, s_{4}$, and $s_{4}$ exhibit a minimum normalized MSPE separately for the given AR parameters of $s_{1}, s_{2}, s_{3}$, and $s_{4}$, as do $s_{3}, s_{4}, s_{3}$, and $s_{4}$ in Table 2 . In other words,

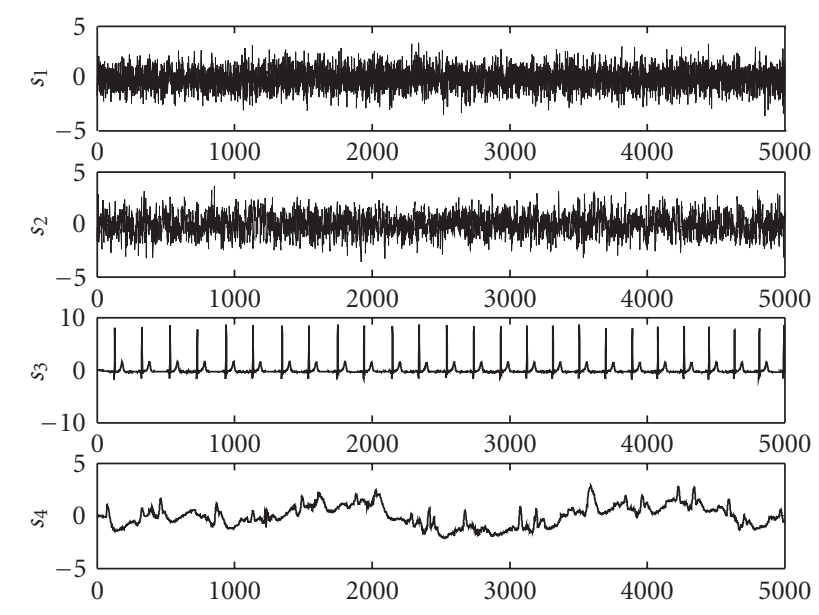

FIgURE 1: Four source signals in simulations.

TABLE 1: The MSPE of different sources for a different given AR parameter $(p=10)$.

\begin{tabular}{lcccc}
\hline & MSPE of $s_{1}$ & MSPE of $s_{2}$ & MSPE of $s_{3}$ & MSPE of $s_{4}$ \\
\hline Given AR of $s_{1}$ & 0.9974 & 0.9559 & $\mathbf{0 . 9 4 5 9}$ & 0.9596 \\
Given AR of $s_{2}$ & 1.9918 & 0.5176 & 0.2581 & $\mathbf{0 . 1 9 3 7}$ \\
Given AR of $s_{3}$ & 48.5098 & 4.8560 & 0.0731 & $\mathbf{0 . 0 3 0 9}$ \\
Given AR of $s_{4}$ & 4.0648 & 0.9520 & 0.1877 & $\mathbf{0 . 0 0 2 5}$ \\
\hline
\end{tabular}

TABLE 2: The MSPE of different sources for a different given AR parameter $(p=20)$.

\begin{tabular}{lcccc}
\hline & MSPE of $s_{1}$ & MSPE of $s_{2}$ & MSPE of $s_{3}$ & MSPE of $s_{4}$ \\
\hline Given AR of $s_{1}$ & 0.9949 & 0.9548 & $\mathbf{0 . 9 4 4 4}$ & 0.9836 \\
Given AR of $s_{2}$ & 1.9875 & 0.5171 & 0.2581 & $\mathbf{0 . 1 9 4 1}$ \\
Given AR of $s_{3}$ & 760.6064 & 76.1407 & $\mathbf{0 . 0 5 0 7}$ & 0.2037 \\
Given AR of $s_{4}$ & 4.0463 & 0.9531 & 0.1899 & $\mathbf{0 . 0 0 2 4}$ \\
\hline
\end{tabular}

when $p=10$ it is possible to extract $s_{3}$ as the first output for the given AR parameters of $s_{1}$, extract $s_{4}$ for $s_{2}$, extract $s_{4}$ for $s_{3}$, and extract $s_{4}$ for $s_{4}$, which means that only $s_{4}$ is the desired signal. When $p=20$, it is possible to extract $s_{3}$ for $s_{1}$, extract $s_{4}$ for $s_{2}$, extract $s_{3}$ for $s_{3}$, and extract $s_{4}$ for $s_{4}$, which means that $s_{3}$ and $s_{4}$ are the desired signals. From this we can conclude that the desired signal does not always have the minimum normalized MSPE among the sources, and that the first extracted signal $[17,19]$ will not always be the desired one.

\section{PROPOSED NEW COST FUNCTION}

Having discussed the issue of MSPE and the first extracted signal, the next problem that must be overcome is how to extract the desired signal for any given AR parameter. To do this we introduce the concept of mean cross prediction error (MCPE). 
TABLE 3: The MCPE of different sources for different given AR parameter $(p=10)$.

\begin{tabular}{lcccc}
\hline & MCPE of $s_{1}$ & MCPE of $s_{2}$ & MCPE of $s_{3}$ & MCPE of $s_{4}$ \\
\hline Given AR of $s_{1}$ & $-\mathbf{0 . 0 0 0 0}$ & 0.5877 & 0.7940 & 0.9578 \\
Given AR of $s_{2}$ & -1.3372 & $\mathbf{0 . 0 0 0 1}$ & 0.1845 & 0.1923 \\
Given AR of $s_{3}$ & -46.4023 & -4.4776 & $\mathbf{0 . 0 0 3 8}$ & 0.0067 \\
Given AR of $s_{4}$ & -2.6264 & -0.2494 & 0.0501 & $\mathbf{0 . 0 0 0 0}$ \\
\hline
\end{tabular}

TABle 4: The MCPE of different sources for different given AR parameter $(p=20)$.

\begin{tabular}{lcccc}
\hline \multicolumn{1}{c}{ MCPE of $s_{1}$} & MCPE of $s_{2}$ & MCPE of $s_{3}$ & MCPE of $s_{4}$ \\
\hline Given AR of $s_{1}$ & $-\mathbf{0 . 0 0 0 2}$ & 0.5861 & 0.7927 & 0.9818 \\
Given AR of $s_{2}$ & -1.3328 & $\mathbf{0 . 0 0 0 1}$ & 0.1844 & 0.1928 \\
Given AR of $s_{3}-749.8778$ & -75.0464 & $\mathbf{0 . 0 0 2 2}$ & -0.1778 \\
Given AR of $s_{4}$ & -2.6062 & -0.2454 & 0.0519 & $\mathbf{0 . 0 0 0 0}$ \\
\hline
\end{tabular}

\subsection{Mean square cross prediction error (MSCPE)}

For given AR model parameters $\mathbf{b}$ of the desired source signal $s_{k}$, the MCPE of each source is expressed as $E\left\{e_{i}(n) e_{j}(n-\right.$ $q)\}(j=1,2, \ldots, m)$, which has the following properties:

$$
\begin{aligned}
E\left\{e_{k}(n) e_{k}(n-q)\right\} & \\
=E\left\{\left(s_{k}(n)-\mathbf{b}^{T} \boldsymbol{S}_{k}(n)\right)\left(s_{k}(n-q)-\mathbf{b}^{T} \boldsymbol{S}_{k}(n-q)\right)\right\} & =0, \\
0<q & \leq p, \\
E\left\{e_{\mathrm{j}}(n) e_{\mathrm{j}}(n-q)\right\} & \\
=E\left\{\left(s_{\mathrm{j}}(n)-\mathbf{b}^{T} \boldsymbol{S}_{\mathbf{j}}(n)\right)\left(s_{\mathrm{j}}(n-q)-\mathbf{b}^{T} \boldsymbol{S}_{\mathbf{j}}(n-q)\right)\right\} & \neq 0, \\
j & \neq k
\end{aligned}
$$

where $q$ denotes the time delay. Thus the sources are divided into two groups: desired and not desired. The MCPE of the desired one is equal to zero, and MCPEs of the others are not. In numerical computation of statistic signals, the above two expressions, (10), will guarantee that the absolute value of MCPE of the desired signal will be smaller than that other signals'.

Reconsidering the benchmarks $s_{1}, s_{2}, s_{3}$, and $s_{4}$ in Section 2, we calculate the corresponding MCPEs for the given AR parameter of different sources, and summarize the results in Table 3 with $p=10$ and in Table 4 with $p=20$. We could see in both Tables 3 and 4 that $s_{1}, s_{2}, s_{3}$, and $s_{4}$ have the minimum absolute MCPE value separately for the given AR parameters of $s_{1}, s_{2}, s_{3}$, and $s_{4}$. Thus the desired source signal has the minimum normalized absolute MCPE value among the sources.

The above analysis urged us to propose a new cost function to solve the problem on how to extract the desired signal for given AR parameters. However, the MCPE is often negative as Tables 3 and 4 did, and thus could not be utilized directly as cost function. Then we introduced the power of $\mathrm{MCPE}$ as cost function.
Looking back to the MCPE of output $y$ can be expressed as $E\{e(n) e(n-q)\}$, the corresponding $e(n)$ is

$$
\begin{aligned}
e(n)= & y(n)-\mathbf{b}^{T} \mathbf{Y}(n) \\
= & \mathbf{w}^{T} \mathbf{x}(n)-\left[b_{1}, b_{2}, \ldots, b_{\mathrm{P}}\right] \\
& \times\left[\mathbf{w}^{T} \mathbf{x}(n-1) \mathbf{w}^{T} \mathbf{x}(n-2), \ldots, \mathbf{w}^{T} \mathbf{x}(n-p)\right]^{T} \\
= & \mathbf{w}^{T} \mathbf{x}(n)-\mathbf{w}^{T} \sum_{i=1}^{p} b_{i} \mathbf{x}(n-i) \\
= & \mathbf{w}^{T}\left(\mathbf{x}(n)-\sum_{i=1}^{p} b_{i} \mathbf{x}(n-i)\right) .
\end{aligned}
$$

With the help of expression (4),e(n) becomes

$$
\begin{aligned}
e(n) & =\mathbf{w}^{T} z(n)=z(n)^{T} \mathbf{w}, \\
e(n-q) & =\mathbf{w}^{T} z(n-q)=z^{T}(n-q) \mathbf{w}, \\
E\{e(n) e(n-q)\} & =E\left\{\mathbf{w}^{T} z(n) z^{T}(n-q) \mathbf{w}\right\} \\
& =\mathbf{w}^{T} E\left\{z(n) z^{T}(n-q)\right\} \mathbf{w} .
\end{aligned}
$$

Furthermore, denote

$$
\mathbf{Z}(q)=E\left\{z(n) z^{T}(n-q)\right\}
$$

and the MCPE is described as

$$
E\{e(n) e(n-q)\}=\mathbf{w}^{T} \mathbf{Z}(q) \mathbf{w} .
$$

Thus we propose the mean square cross prediction error (MSCPE), expressed as $\mathbf{w}^{T} \mathbf{Z}(q) \mathbf{Z}^{T}(q) \mathbf{w}^{T}$, as a new cost function under the constraint $\mathbf{w}^{T} \mathbf{w}=1$ to solve the above problem. The cost function in a simple form is

$$
\begin{gathered}
J_{q}(\mathbf{w})=\mathbf{w}^{T} \mathbf{Z}(q) \mathbf{Z}^{T}(q) \mathbf{w}, \quad 0<q \leq p \\
\text { s.t. } \mathbf{w}^{T} \mathbf{w}=1
\end{gathered}
$$

If the sources have different AR model parameters, MSCPE will have only one minimum, that is, zero, for specific AR parameter. Thus we can extract any desired signal by minimizing the cost function $J(\mathbf{w})$.

\subsection{Algorithm using EVD}

Note that the above expression (15) implies that the minimization of the cost function $J(\mathbf{w})$ under the constraint $\mathbf{w}^{T} \mathbf{w}=1$ is equivalent to finding the eigenvector corresponding to the minimal eigenvalue of the real symmetric matrix $\mathbf{Z}(q) \mathbf{Z}^{T}(q)$. Moreover, $\mathbf{w}$ is equivalent to the singular vector of the minimal singular value of $\mathbf{Z}(q)$. Thus $\mathbf{w}$ can be calculated using the following method:

$$
\begin{aligned}
z(n) & =\left(x(n)-\sum_{\mathrm{i}=1}^{p} b_{\mathrm{i}} \mathbf{x}(n-i)\right), \\
\mathbf{Z}(q) & =E\left\{z(n) z^{T}(n-q)\right\}, \\
\mathbf{w} & =\operatorname{MinEVD}\left\{\mathbf{Z}(q) \mathbf{Z}^{T}(q)\right\}=\operatorname{MinSVD}\{\mathbf{Z}(q)\},
\end{aligned}
$$


where $\operatorname{MINEVD}\{T\}$ is an operator that calculates the normalized eigenvector corresponding to the minimal eigenvalue of the real symmetric matrix $T$ and $\operatorname{MINSVD}\{T\}$ is an operator that calculate the normalized singular vector corresponding to the minimal singular value of the matrix $T$.

\subsection{Solving the permutation problem}

BSS has the drawbacks of permutation problem. Then it has to be verifed that the proposed algorithm can extract the desired signal as the first output. If we have known the AR model parameters of one desired signal, Theorem 1 shows that the algorithm given in expression (16) will avoid the permutation problem and can extract the target source.

Theorem 1. Define performance vector $c=\mathbf{A}^{T} \mathbf{w}_{*}$, where $\mathbf{w}_{*}$ is the vector of weights estimated using the proposed algorithm

$$
\begin{gathered}
\mathbf{w}_{*}=\operatorname{MINEVD}\left\{\mathbf{Z}(q) \mathbf{Z}^{T}(q)\right\}=\operatorname{MINSVD}\{\mathbf{Z}(q)\} \\
\left\|\mathbf{w}^{*}\right\|^{2}=1 .
\end{gathered}
$$

If for the specific AR model parameters of desired signal $s_{k}$, expressions (10) both hold, then $c=\beta \varepsilon_{k}$, where $\beta$ is a nonzero scalar and $\varepsilon_{k}=[0, \ldots, 1, \ldots, 0]^{T}$ is a basis vector, that is, the $k$ th element equals 1 .

Proof. Considering the objective function $J(w)$, we had

$$
\begin{gathered}
J(\mathbf{w})=\mathbf{w}_{*}^{T} \mathbf{Z}(q) \mathbf{Z}^{T}(q) \mathbf{w}_{*} \\
=\mathbf{w}_{*}^{T} E\left\{z(n) z^{T}(n-q)\right\} E^{T}\left\{z(n) z^{T}(n-q)\right\} \mathbf{w}_{*} \\
=\mathbf{w}_{*}^{T} \mathbf{A} E\left\{\left(\mathbf{s}(n)-\sum_{i=1}^{p} b_{\mathrm{i}} \mathbf{s}(n-i)\right)\right. \\
\left.\quad \times\left(\mathbf{s}(n-q)-\sum_{i=1}^{p} b_{\mathrm{i}} \mathbf{s}(n-i-q)\right)\right\}, \\
E^{T}\left\{\left(\mathbf{s}(n)-\sum_{i=1}^{p} b_{\mathrm{i}} \mathbf{s}(n-i)\right)\right. \\
\left.\quad \times\left(\mathbf{s}(n-q)-\sum_{i=1}^{p} b_{\mathrm{i}} \mathbf{s}(n-i-q)\right)\right\} \mathbf{A}^{T} \mathbf{w}_{*} \\
=\mathbf{w}_{*}^{T} \mathbf{A} \boldsymbol{\Phi} \boldsymbol{\Phi}^{T} \mathbf{A}^{T} \mathbf{w}_{*}=c^{T} \mathbf{\Phi} \boldsymbol{\Phi}^{T} \mathbf{A} c,
\end{gathered}
$$

where the entries of $\Phi$ are denoted by $\Phi_{i j}$ :

$$
\begin{aligned}
\Phi_{i j} & =E\left\{e_{i}(n) e_{j}(n-q)\right\}, \\
e_{i}(n) & =s_{i}(n)-\sum_{i=1}^{p} b_{i} s_{j}(n-i), \\
e_{j}(n-q) & =s_{j}(n-q)-\sum_{i=1}^{p} b_{i} s_{j}(n-i-q) .
\end{aligned}
$$

Since $E\left\{\underline{\mathbf{s s}}^{T}\right\}=\mathbf{I}, E\left\{e_{k}(n) e_{k}(n-q)\right\}=0$, and $E\left\{e_{j}(n) e_{j}(n-\right.$ $q)\} \neq 0, j \neq k$, the expression (19) can be rewritten as

$$
\Phi_{i j} \begin{cases}=0, & i \neq j, \\ =0, & i=j=k \\ \neq 0, & i=j \neq k .\end{cases}
$$

Denote by $\boldsymbol{\Psi}=\boldsymbol{\Phi} \boldsymbol{\Phi}^{T}$, and then $\boldsymbol{\Psi}$ will be diagonal. $\Psi_{j j}$ has its element null only when $j$ is equal to $k$. Thus the minimum for $J(\mathbf{w})$, besides the trivial $c=0$ (which is not allowed by normalization) is $c=\beta \varepsilon_{k}$.

Theorem 1 shows that this cost function embodied the desired property. Moreover, if we randomly generate AR model parameters, we will extract a signal with minimal MSCPE.

\section{SIMULATIONS ON BENCHMARK DATA}

Extensive computer simulations and experiments have been performed to verify the proposed algorithm. In the simulations, suppose that the AR model parameter of the desired signal is known, and algorithm (16) will be used to extract the desired one.

For comparisons, denoted the algorithm in [19] by ALG1; fast-ICA algorithm [22] by ALG2; and our algorithm (16) with $q=1$ by Ours1. ALG1 utilizes the MSPE as cost function with ordinary gradient descent method, while Ours1 utilizes the MSCPE as the cost function.

In this section, four experiments (Exps) are performed to demonstrate the validity of our algorithm, and comparisons are done between ALG1 and Ours1. We still utilize the four benchmarks $s_{1}, s_{2}, s_{3}$, and $s_{4}$ in Figure 1. The four signals would be extracted respectively in separate experiments. The elements of the mixing matrix are randomly generated according to the Gaussian distribution with zero mean and unit variance. After prewhitening, $E\left\{\mathbf{x x}^{T}\right\}=\mathbf{I}$ and $y=$ $\mathbf{w}^{T} \mathbf{x}=\mathbf{w}^{T}$ As.

For each desired signal $s_{k}(k=1,2,3,4)$ with its AR model parameters $\mathbf{b}$, the MSPE $E^{2}\left\{e_{i}^{2}(n)\right\}(i=1,2,3,4)$ is expressed as

$$
E\left\{e_{i}^{2}(n)\right\}=E\left\{\left(s_{i}(n)-b^{T} \mathbf{S}_{i}(n)\right)^{2}\right\}, \quad i=1,2,3,4
$$

and its MSCPE $E^{2}\left\{e_{i}(n) e_{i}(n-q)\right\}(i=1,2,3,4)$ with $q=1$ is

$$
\begin{array}{r}
E^{2}\left\{e_{i}(n) e_{i}(n-1)\right\} \\
=E^{2}\left\{\left(s_{i}(n)-b^{T} S_{i}(n)\right)\left(s_{i}(n-1)-b^{T} S_{i}(n-1)\right)\right\} \\
i=1,2,3,4
\end{array}
$$

Furthermore, the following performance index (PI) will be adopted to measure the extracted signal:

$$
\mathrm{PI}(i)=10 \log _{10}\left(\frac{1}{m-1}\left(\sum_{j=1}^{l} \frac{\left|g_{j}\right|^{2}}{\max _{i}\left|g_{i}\right|^{2}}-1\right)\right),
$$


TABle 5: Simulations with known AR parameters $(p=20)$.

\begin{tabular}{|c|c|c|c|c|c|c|c|}
\hline & $\begin{array}{c}\text { Desired signal } \\
\left(s_{k}\right)\end{array}$ & $\begin{array}{l}\text { Signal }\left(s_{k}\right) \text { with } \\
\text { mininmal MSPE }\end{array}$ & $\begin{array}{l}\text { Signal }\left(s_{k}\right) \text { with } \\
\text { mininmal MSCPE }\end{array}$ & $\begin{array}{c}\text { Extracted signal } \\
\left(s_{k}\right) \text { by ALG1 }\end{array}$ & $\begin{array}{c}\text { Extracted signal } \\
\left(s_{k}\right) \text { by Ours } 1\end{array}$ & PI of ALG1 (dB) & $\begin{array}{c}\text { PI of Ours] } \\
(\mathrm{dB})\end{array}$ \\
\hline $\operatorname{Exp}_{1}$ & $s_{1}$ & $s_{3}$ & $s_{1}$ & $s_{3}$ & $s_{1}$ & -35.80 & -32.12 \\
\hline $\operatorname{Exp}_{2}$ & $s_{2}$ & $s_{4}$ & $s_{2}$ & $s_{4}$ & $s_{2}$ & -33.25 & -35.32 \\
\hline $\operatorname{Exp}_{3}$ & $s_{3}$ & $s_{3}$ & $s_{3}$ & $s_{3}$ & $s_{3}$ & -32.56 & -43.68 \\
\hline $\operatorname{Exp}_{4}$ & $s_{4}$ & $s_{4}$ & $s_{4}$ & $s_{4}$ & $s_{4}$ & -35.45 & -41.40 \\
\hline
\end{tabular}

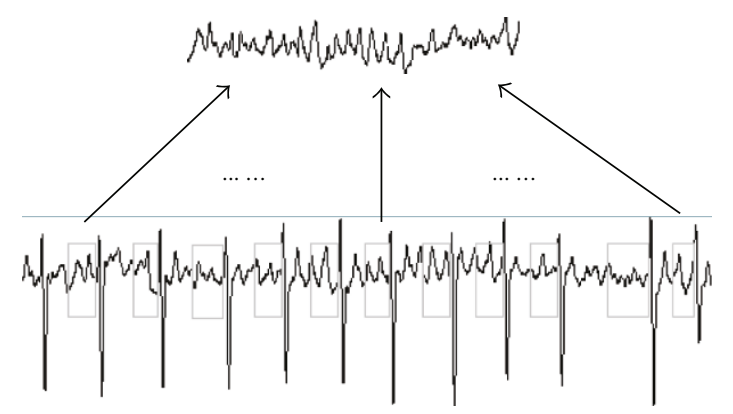

Figure 2: Generation of rough AA waves from V1 lead in an AF episode.

where $g_{i}$ is the $i$ th element of the global system vector $\mathbf{g}=$ $\mathbf{w}^{T} \mathbf{A}$, which is normalized as $\mathbf{g}^{T} \mathbf{g}=1$, and $m$ is equal to four. Generally speaking, an algorithm will work well if PI is less than $-30 \mathrm{~dB}$.

In each experiment $\left(\operatorname{Exp}_{i} i=1,2,3,4\right)$, the AR model parameters $\mathbf{b}$ of $s_{i}(i=1,2,3,4)$ are got using Matlab function "aryule" with a length of $p=20$. The results are obtained by averaging over 100 relisations, and are summarized in Table 5, which shows that ALG1 extracts the signal with minimal MSPE, which corresponds to the analysis of Table 2, and Ours1 extracts the one with minimal MSCPE; meanwhile, the signal with minimal MSCPE corresponds to the desired one in all experiments while MSPE only does in the third and fourth experiments.

As a result, the two algorithms can both extract original signal on the viewpoint of extracting an arbitrary signal, but Ours1 works better than ALG1 on the viewpoint of extracting a desired signal. It results from the fact in Tables 1-4 that the desired signal has the minimal MSCPE but not always the MSPE.

\section{ESTIMATING THE AR MODEL PARAMETERS OF AA SOURCE}

The above algorithm turns the problem of how to extract a desired signal into that of how to estimate the corresponding AR parameters. Then we focused the research on how to estimate AA signal with its estimated AR parameters.

Note that Castells et al. $[8,10]$ have obtained the fibrillatory wave from AF recordings by linking together consecutive T-Q segments with no VA to synthesize AF signal. The generation of synthesized AF ECG casts lights upon our research. Since R wave will be easily recognized by computer for its peak while $\mathrm{T}$ wave is not, and $\mathrm{Q}$ wave

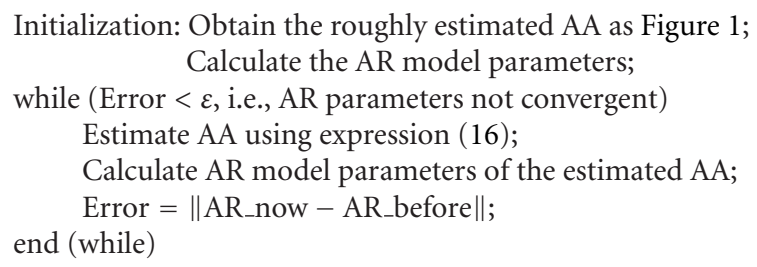

Algorithm 1

neighbours $\mathrm{R}$ wave, we select the later half samples of $\mathrm{R}-\mathrm{R}$ intervals instead of T-Q intervals to generate the rough AA and estimated its AR parameters. In order to smooth the transitions between different intervals, we employed cubic spline interpolation. Furthermore, clinical experience shows that V1 lead contains the largest AA contribution among the 12-lead surface ECGs, which has been confirmed by Rieta et al. [7] using ICA or BSS methods. Thus we would like to select V1 lead to execute the above methods. Figure 2 illustrates how rough AA can be created from AF episode.

Initially, the coarse AR parameters are obtained using the rough AA signal. And they would be substituted into expression (16) to extract AA signal. Then fine AR parameters would be obtained. This calculation will not stop until the AR parameters are convergent. Accordingly, the approaches to extract AA in AF can be summarized as in Algorithm 1 where the operator $\|\cdot\|$ denotes the Frobenius norm and $\varepsilon$ is the truncation error.

\section{EXPERIMENTS ON REAL-WORLD 12-LEAD ELECTROCARDIOGRAM (ECG) IN ATRIAL FIBRILLATION (AF)}

The simulation in Section 4 shows that the desired signal could be extracted if its AR parameters have been known, and verifies expression (16). If the AR parameters might not been known in AA case, AA source would be extracted from real-world ECGs using Algorithm 1. For convenience, denote Algorithm 1 with $q=1$ by Ours2.

Since AA signal has a main peak between 3.5 and $10 \mathrm{~Hz}$, and all nonrelated AA components, such as VA, have other important spectral contents outside the band of the peak; the spectral concentration (SC) $[8,10]$ around the main frequency peak $f_{p}$ can be used as an indicator to measure the quality of the estimated AA in real AF signal. High 


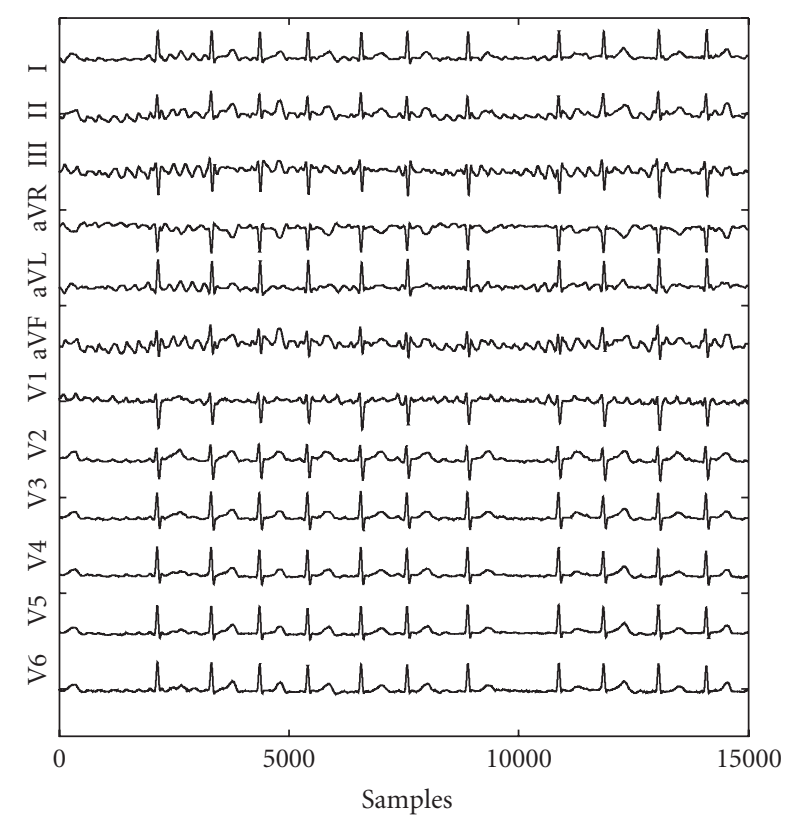

FIgUre 3: A 12-lead ECG segment from a patient in AF used as a learning set.

spectral concentration values in the band of peak indicate high quality in AA estimation. SC is defined as

$$
\mathrm{SC}=\frac{\sum_{0.82 f p}^{1.17 f p} P_{\mathrm{AA}}\left(f_{i}\right)}{\sum_{0}^{f_{s / 2}} P_{\mathrm{AA}}\left(f_{i}\right)} \times 100 \%,
$$

where $P_{\mathrm{AA}}$ is the power spectrum of the AA signal.

In this experiment, twelve ECGs from eight patients in persistent AF are tested to extract AA signal. Each lead in every ECG contains 15000 samples (1920 samples per second) with 16-bit amplitude. Figure 3 shows ECGs from Patient $_{1}$. In order to validate the AA identification, the power spectral density (PSD) is computed for all of the extracted signals. The procedure consists of periodogram method with a rectangular window of 2048 points length, a $50 \%$ overlapping between adjacent windowed section, and an 8192-point fast Fourier transform (FFT). AR model parameters are calculated using Matlab function "aryule" with a length of $p=200$. AA has a main frequency peak around $3.5-10 \mathrm{~Hz}$, which is called region of interest (ROI). Generally speaking, if the signal extracted from surface ECGs both has a main peak between 3.5 and $10 \mathrm{~Hz}$ and has an SC of more than $40 \%$, it could be regarded as AA signal.

Figure 4(b) plots one extracted AA from ECG $/$ Patient $_{1}$, and Figure 4(a) shows the corresponding PSD along with the atrial frequency, where the spectral content above $2 \mathrm{~Hz}$ is discarded due to its low contribution. The extracted one can be regarded as AA signal, for that it has only one peak frequency in ROI, and its SC is more than $40 \%$.

Comparisons are done among ALG1, ALG2, and Ours2 algorithms, and the AR parameters used in ALG1 would be obtained from the extracted AA by ALG2. The applications of the three algorithms on the twelve real ECGs are summarized

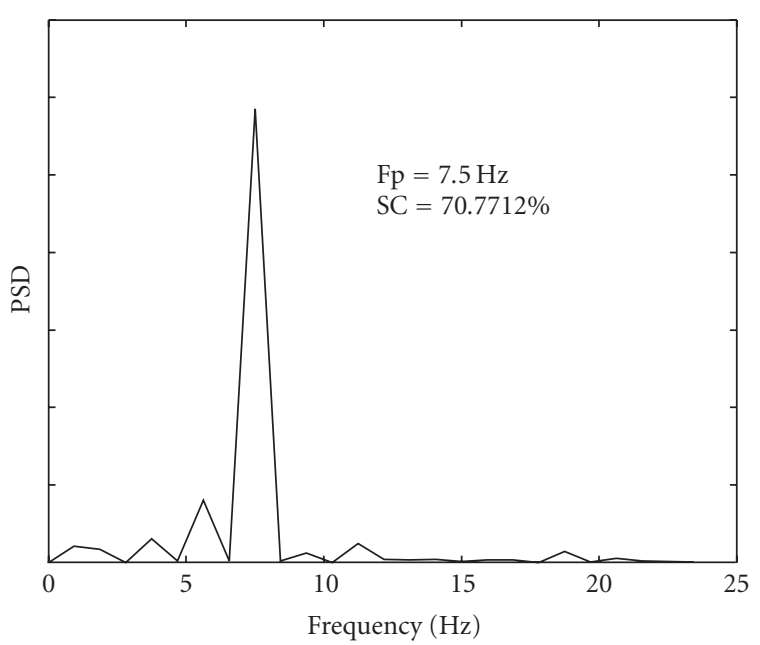

(a)

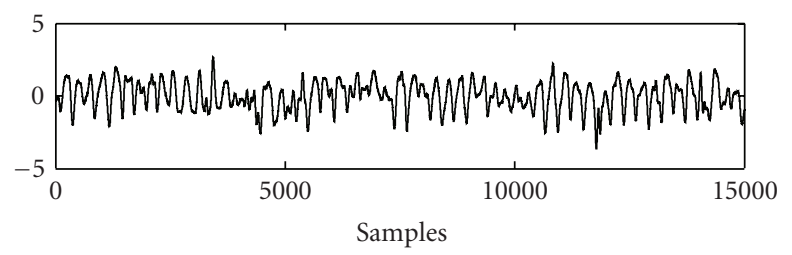

(b)

Figure 4: (a) The extracted AA signal from patient one; (b) The corresponding PSD along with the atrial frequency.

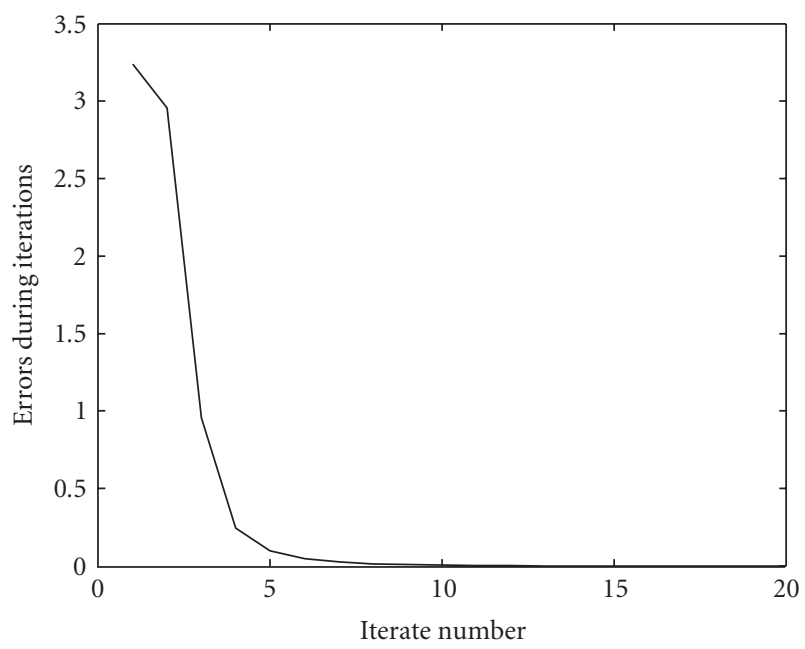

FIgURE 5: The learning curve of Algorithm 1.

in Table 6. It shows that none of the signals extracted by ALG1 has an SC of more than 40\%. The main frequencies of atrial wave extracted by Ours2 range from 4.7 to $8.4 \mathrm{~Hz}$, and the SC is $53.97 \%$ on average. The main frequencies extracted by ALG2 are from 4.7 to $8.4 \mathrm{~Hz}$, and the SC is $50.91 \%$ on average. These results demonstrate that the proposed algorithm is as efficient as ICA method, but the algorithm based on linear predictor fails to extract AA signal. Figure 5 plots the averaged learning curve of Ours2 for the twelve extractions. All of the twelve extractions are converged in 
TABle 6: Experiments of ALG1, ALG2, and Ours2.

\begin{tabular}{|c|c|c|c|c|c|c|}
\hline $\mathrm{ECG}_{i} /$ Patient $_{j}$ & SC of ALG1 (\%) & SC of ALG2 (\%) & SC of Ours2 (\%) & Fp of ALG1 $(\mathrm{Hz})$ & Fp of ALG2 $(\mathrm{Hz})$ & Fp of Ours2 $(\mathrm{Hz})$ \\
\hline$i=1 / j=1$ & 24.8519 & 66.8203 & 70.7712 & 4.2353 & 7.5000 & 7.5000 \\
\hline$i=2 / j=1$ & 25.9875 & 58.7091 & 62.1591 & 2.8125 & 7.5000 & 7.5000 \\
\hline$i=3 / j=2$ & 19.1397 & 51.0525 & 50.4415 & 1.8750 & 5.6250 & 5.6250 \\
\hline$i=4 / j=2$ & 23.2529 & 50.7547 & 52.2671 & 3.7500 & 7.5000 & 7.5000 \\
\hline$i=5 / j=3$ & 16.6486 & 55.9363 & 59.9669 & 0.9375 & 4.6875 & 4.6875 \\
\hline$i=6 / j=3$ & 16.7755 & 57.9015 & 59.3211 & 1.8750 & 4.6875 & 4.6875 \\
\hline$i=7 / j=4$ & 16.1763 & 48.4142 & 46.8586 & 2.8125 & 6.5625 & 6.5625 \\
\hline$i=8 / j=4$ & 10.4663 & 38.1165 & 44.5730 & 3.7500 & 6.5625 & 6.5625 \\
\hline$i=9 / j=5$ & 22.3358 & 44.7337 & 48.2786 & 1.8750 & 8.4375 & 8.4375 \\
\hline$i=10 / j=6$ & 32.3524 & 43.1452 & 46.6405 & 3.7500 & 7.5000 & 7.5000 \\
\hline$i=11 / j=7$ & 14.2147 & 47.3161 & 55.4149 & 0.9375 & 4.6875 & 4.6875 \\
\hline$i=12 / j=8$ & 14.9846 & 48.0524 & 59.1223 & 1.8750 & 5.6250 & 5.6250 \\
\hline Mean & 19.7655 & 50.9127 & 54.6513 & 2.5404 & 6.4063 & 6.4063 \\
\hline
\end{tabular}

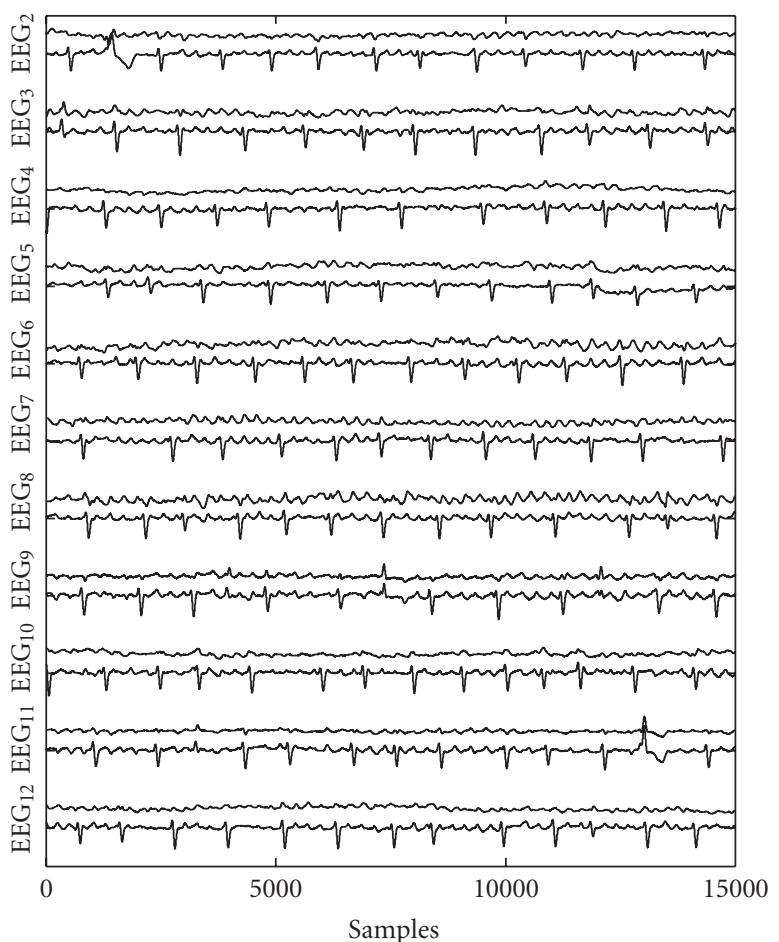

Figure 6: AA extraction results from $\mathrm{ECG}_{2}-\mathrm{ECG}_{12}$. It shows the extracted AA source (top) and lead V1 (bottom).

ten iterations. Moreover, the BSE-based algorithm needs less computation than ICA.

In the twelve ECGs, the former eight are divided into four different groups, which come from four different patients, and each group contains two ECGs. The extracted results in Table 6 show that different ECGs from the same patient (e.g., patient ${ }_{2}$ ) sometimes have different main frequencies, which verifies that $\mathrm{AA}$ is nonstationary in long term.

The visual comparisons between the extracted signals and the AA present in the original ECG are summarized in Figure 6, which provided satisfactory results. These results

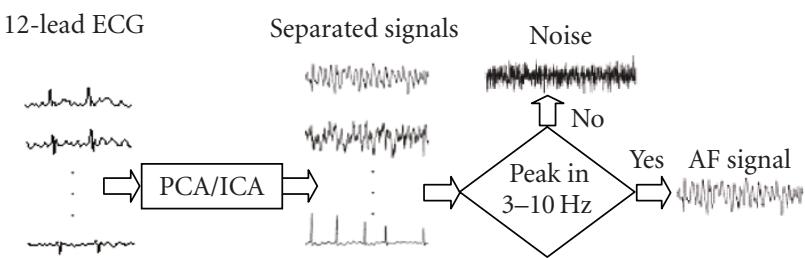

Figure 7: The frame based on ICA or PCA.

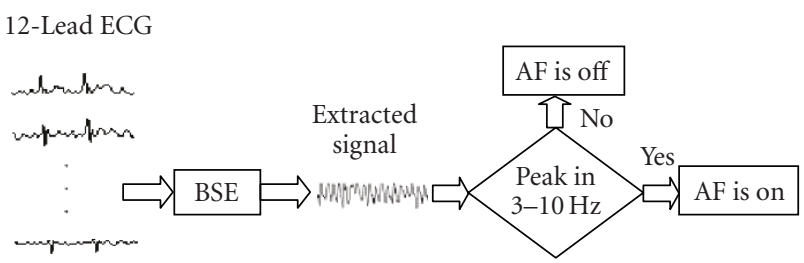

FIGURE 8: The new frame based on BSE.

correspond to $\mathrm{ECG}_{2}-\mathrm{ECG}_{12}$. In the figure, lead V1 (in the bottom) can be observed from the 12-lead ECG in AF, along with the Ours2-estimated AA for that episode (at the top) for visual comparison. The estimated AA has been scaled by the factor associated with its projection onto lead V1.

\section{DISCUSSION AND CONCLUSIONS}

In this paper, we propose an efficient semi-BSE algorithm based on AR model parameters to extract a specific signal. The algorithm transforms the problem on how to extract a specific signal into that on how to estimate its AR model, and is verified by the theoretical analysis and computer simulations. The algorithm embodies the desired property, and it can be used in related fields where the AR parameters of the desired signal can be approximately estimated before extraction. On this standpoint this algorithm is more robust than the ones based on linear predictor [19]. Moreover, this algorithm can be regarded as an extension of the algorithm in [19], when the time delay $q$ is equal to zero. 
The expression (16) showed that the proposed algorithm relates to minor component analysis (MCA). Thus, many results $[11,23]$ on MCA can be used to improve the algorithm, which will be our future work.

From the methodological standpoint, we propose another BSE-based algorithm to extract AA signal except primary component analysis (PCA), ICA, and spatiotemporal QRST cancellation (STC) methods [5].

STC techniques obtained as many AA signals as leads processed by the cancellation algorithm. In contrast, the ICA/PCA-based approaches estimate a single signal, which is able to reconstruct the complete AA present in every ECG lead, thus the two methods are more robust than STC method. Figure 7 describes the frame of ICA/PCA method, and shows that ICA/PCA method cannot directly extract the AF signal; power spectrum analysis will be utilized to tell which one is AF signal by judging whether the signal has a peak in $3-10 \mathrm{~Hz}$. As a contrast, the BSE-based frame shown in Figure 8 [24] only extracts one signal, thus it has lower computational load than Figure 7, need only onetwelfth memory of ICA/PCA method, and it is more suitable in clinical monitoring. The proposed algorithm could be applied into this frame and will have great potential in clinical monitoring machine.

\section{ACKNOWLEDGMENTS}

This work is supported by National Nature Science Foundation 60571047. The authors wish to thank the National project for postgraduates of key constructed high-level universities in China in 2007.

\section{REFERENCES}

[1] J. S. Steinberg, S. Zelenkofske, S.-C. Wong, M. Gelernt, R. Sciacca, and E. Menchavez, "Value of the P-wave signalaveraged ECG for predicting atrial fibrillation after cardiac surgery," Circulation, vol. 88, no. 6, pp. 2618-2622, 1993.

[2] R. G. Tieleman, I. C. Van Gelder, H. J. G. M. Crijns, et al., "Early recurrences of atrial fibrillation after electrical cardioversion: a results of fibrillation-induced electrical remodeling of the atria?" Journal of the American College of Cardiology, vol. 31, no. 1, pp. 167-173, 1998.

[3] O. D. Escoda, L. Granai, M. Lemay, J. M. Hernandez, P. Vandergheynst, and J.-M. Vesin, "Ventricular and atrial activity estimation through sparse ECG signal decompositions," in Proceedings of the IEEE International Conference on Acoustics, Speech and Signal Processing (ICASSP '06), vol. 2, pp. 1060 1063, Toulouse, France, May 2006.

[4] J. J. Rieta and F. Hornero, "Comparative study of methods for ventricular activity cancellation in atrial electrograms of atrial fibrillation," Physiological Measurement, vol. 28, no. 8, pp. 925-936, 2007.

[5] M. Lemay, V. Jacquemet, A. Forclaz, J. M. Vesin, and L. Kappenberger, "Spatiotemporal QRST cancellation method using separate QRS and T-waves templates," in Proceedings of Computers in Cardiology, pp. 611-614, Lyon, France, September 2005.

[6] J. J. Rieta, F. Hornero, C. Sánchez, C. Vayá, D. Moratal, and J. M. Sanchis, "Derivation of atrial surface reentries applying ICA to the standard electrocardiogram of patients in postoperative atrial fibrillation," in Proceedings of the 6th
International Conference on Independent Component Analysis and Blind Signal Separation (ICA '06), vol. 3889, pp. 478-485, Charleston, SC, USA, March 2006.

[7] J. J. Rieta, F. Castells, C. Sánchez, V. Zarzoso, and J. Millet, "Atrial activity extraction for atrial fibrillation analysis using blind source separation," IEEE Transactions on Biomedical Engineering, vol. 51, no. 7, pp. 1176-1186, 2004.

[8] F. Castells, J. J. Rieta, J. Millet, and V. Zarzoso, "Spatiotemporal blind source separation approach to atrial activity estimation in atrial tachyarrhythmias," IEEE Transactions on Biomedical Engineering, vol. 52, no. 2, pp. 258-267, 2005.

[9] P. Langley, J. J. Rieta, M. Stridh, J. Millet, L. Sornmo, and A. Murray, "Comparison of atrial signal extraction algorithms in 12-lead ECGs with atrial fibrillation," IEEE Transactions on Biomedical Engineering, vol. 53, no. 2, pp. 343-346, 2006.

[10] F. Castells, J. Igual, J. Millet, and J. J. Rieta, "Atrial activity extraction from atrial fibrillation episodes based on maximum likelihood source separation," Signal Processing, vol. 85, no. 3, pp. 523-535, 2005.

[11] A. Cichocki and S. B. Amari, Adaptive Blind Signal and Image Processing: Learning Algorithms and Applications, John Wiley \& Sons, Chichester, UK, 2002.

[12] A. Hyvärinen, J. Karhunen, and E. Oja, Independent Component Analysis, John Wiley \& Sons, Chichester, UK, 2001.

[13] Z.-L. Zhang and Z. Yi, "Robust extraction of specific signals with temporal structure," Neurocomputing, vol. 69, no. 7-9, pp. 888-893, 2006.

[14] W. Lu and J. C. Rajapakse, "Approach and applications of constrained ICA," IEEE Transactions on Neural Networks, vol. 16, no. 1, pp. 203-212, 2005.

[15] A. Cichocki, R. Thawonmas, and S. Amari, "Sequential blind signal extraction in order specified by stochastic properties," Electronics Letters, vol. 33, no. 1, pp. 64-65, 1997.

[16] A. K. Barros and A. Cichocki, "Extraction of specific signals with temporal structure," Neural Computation, vol. 13, no. 9, pp. 1995-2003, 2001.

[17] W. Liu, D. P. Mandic, and A. Cichocki, "Blind second-order source extraction of instantaneous noisy mixtures," IEEE Transactions on Circuits and Systems II, vol. 53, no. 9, pp. 931935, 2006.

[18] A. Cichocki and R. Thawonmas, "On-line algorithm for blind signal extraction of arbitrarily distributed, but temporally correlated sources using second order statistics," Neural Processing Letters, vol. 12, no. 1, pp. 91-98, 2000.

[19] W. Liu, D. P. Mandic, and A. Cichocki, "Blind source extraction based on a linear predictor," IET Signal Processing, vol. 1, no. 1, pp. 29-34, 2007.

[20] M. Holm, S. Pehrson, M. Ingemansson, et al., "Non-invasive assessment of the atrial cycle length during atrial fibrillation in man: introducing, validating and illustrating a new ECG method," Cardiovascular Research, vol. 38, no. 1, pp. 69-81, 1998.

[21] P. Langley, J. P. Bourke, and A. Murray, "Frequency analysis of atrial fibrillation," in Proceedings of Computers in Cardiology, pp. 65-68, Cambridge, Mass, USA, September 2000.

[22] A. Hyvärinen, "Fast and robust fixed-point algorithms for independent component analysis," IEEE Transactions on Neural Networks, vol. 10, no. 3, pp. 626-634, 1999.

[23] E. Oja, "Principal components, minor components, and linear neural networks," Neural Networks, vol. 5, no. 6, pp. 927-935, 1992.

[24] G. Wang, N. Rao, and Y. Zhang, "Atrial fibrillatory signal estimation using blind source extraction algorithm based on high-order statistics," to appear in Science in China, Series F. 